\title{
Non-inflammatory destructive periodontal disease: a clinical, microbiological, immunological and genetic investigation
}

\author{
Carlos Eduardo REPEKE ${ }^{1}$, Cristina Ribeiro CARDOSO ${ }^{2}$, Marcela CLAUDINO ${ }^{3}$, Elcia Maria SILVEIRA ${ }^{1}$, Ana Paula Favaro \\ TROMBONE ${ }^{4}$, Ana Paula CAMPANELLI ${ }^{5}$, João Santana SILVA ${ }^{6}$, Walter MARTINS JÚNIOR ${ }^{7}$, Gustavo Pompermaier \\ GARLET $^{8}$
}

\author{
1- DDS, MSc, PhD student, Department of Biological Sciences, Bauru School of Dentistry, University of São Paulo, Bauru, SP, Brazil. \\ 2- DDS, MSc, PhD, Associated Professor, Department of Clinical Analyses Toxicology Bromatologics, Ribeirão Preto College of Pharmaceutical Sciences, \\ University of São Paulo, Ribeirão Preto, SP, Brazil \\ 3- DDS, MSc, PhD, Latin America Institute for Dental Research and Education-ILAPEO, Curitiba, PR, Brazil. \\ 4- PharmD, MSc, PhD, Department of Biochemistry and Immunology, School of Medicine of Ribeirão Preto, University of São Paulo, Ribeirão Preto, SP, Brazil. \\ 5- ScD, MSc, PhD, Professor, Department of Biological Sciences, Bauru School of Dentistry, University of São Paulo, Bauru, SP, Brazil. \\ 6- ScD, MSc, PhD, Full Professor, Department of Biochemistry and Immunology, School of Medicine of Ribeirão Preto, University of São Paulo, Ribeirao \\ Preto, SP, Brazil. \\ 7- DDS, MSc, PhD, Associate Professor, Department of Periodontics, Dentistry School, University of Ribeirão Preto, Ribeirao Preto, SP, Brazil. \\ 8- DDS, MSc, PhD, Associate Professor, Department of Biological Sciences, Bauru School of Dentistry, University of São Paulo, Bauru, SP, Brazil.
}

Corresponding address: Gustavo P. Garlet - Faculdade de Odontologia de Bauru - USP - Departmento de Ciências Biológicas - Al. Octávio Pinheiro Brisola, 9-75 - 17012-901 - Bauru - SP - Brazil - Phone: +55 (14) 3235-8274 - Fax: +55 (14) 3235-8274 - e-mail: garletgp@usp.br

Received: October 15, 2009 - Modification: February 16, 2010 - Accepted: April 12, 2010

\section{ABSTRACT}

\begin{abstract}
Deriodontitis comprises a group of multifactorial diseases in which periodontopathogens accumulate in dental plaque and trigger host chronic inflammatory and immune responses against periodontal structures, which are determinant to the disease outcome. Although unusual cases of non-inflammatory destructive periodontal disease (NIDPD) are described, their pathogenesis remains unknown. A unique NIDPD case was investigated by clinical, microbiological, immunological and genetic tools. The patient, a non-smoking dental surgeon with excessive oral hygiene practice, presented a generalized bone resorption and tooth mobility, but not gingival inflammation or occlusion problems. No hematological, immunological or endocrine alterations were found. No periodontopathogens ( $A$. actinomycetemcomitans, $P$. gingivalis, $F$. nucleatum and $T$. denticola) or viruses (HCMV, EBV-1 and HSV-1) were detected, along with levels of IL- $1 \beta$ and TNF- $\alpha$ in GCF compatible with healthy tissues. Conversely ALP, ACP and RANKL GCF levels were similar to diseased periodontal sites. Genetic investigation demonstrated that the patient carried some SNPs, as well HLA-DR4 (*0404) and HLA-B27 alleles, considered risk factors for bone loss. Then, a less vigorous and diminished frequency of toothbrushing was recommended to the patient, resulting in the arrest of alveolar bone loss, associated with the return of ALP, ACP and RANKL in GCF to normality levels. In conclusion, the unusual case presented here is compatible with the previous description of NIDPD, and the results that a possible combination of excessive force and frequency of mechanical stimulation with a potentially bone loss prone genotype could result in the alveolar bone loss seen in NIDPD.
\end{abstract}

Key words: Periodontal diseases. Non-inflammatory destructive periodontal

\section{INTRODUCTION}

Mechanical stimulation by toothbrushing is capable of inducing keratinization of the oral sulcular epithelium ${ }^{8}$, enhance gingival circulation ${ }^{50}$, cause formation of procollagen synthesis of gingival fibroblasts, and increase alveolar bone ${ }^{53}$. In addition, it has been shown that proliferation of basal cells, fibroblasts and synthesis of procollagen I are promoted more effectively by mechanical stimulation with a toothbrush than by removal of dental plaque ${ }^{24}$. 
Conversely, a very interesting report described two cases of severe destructive periodontal disease in which the trauma resulting from aggressive daily oral hygiene was supposed to trigger alveolar bone loss ${ }^{40}$. This non-inflammatory destructive periodontal disease (NIDPD) was characterized by severe loss of attachment and resorption of alveolar bone, in sites where gingival inflammation and pocket formation were not prominent features. Moreover, in these patients antimicrobial therapy was not effective in arresting or slowing the progression of the disease, thus suggesting that bacteria could not be the etiological factor in such cases ${ }^{40}$. In accordance to these findings, the authors concluded that soft tissue trauma resulting from aggressive daily oral hygiene combined with a possible susceptible genetic background could determine the pathobiology of NIDPD. However, until now there is no complete description on the contradictory role of the excessive toothbrushing in the development of periodontal disease with the mechanisms underlying NIDPD, which remain to be elucidated.

Accordingly, in the present study we reported a unique detailed NIDPD case study, involving microbiological, immunological and genetic analysis, in which pathologic bone loss was associated to inappropriate toothbrushing.

\section{CASE REPORT}

The patient, a healthy Caucasian 46-year-old lady, dental surgeon, presenting normal weight and regular physical activities, from the southeastern region of Brazil, promptly identified the beginning of teeth mobility and gingival recession on her own mouth, and looked for periodontal health care. First, she was unsuccessfully advised to intensify toothbrushing procedures as an attempt to control the bone resorption. However, in spite of intense toothbrushing and relevant oral hygiene, the gingival recession and teeth mobility were continued and the patient came to evaluation by a periodontist from our research group. The history related by the patient, in this circumstance a dentist, suggested an intriguing case of periodontal disease without visible signs of local inflammation. Then, we investigated this unique condition by performing a complete evaluation of the patient to provide the pathology's diagnosis, its mechanisms of development and to establish the most suitable treatment for this case.

The patient denied any pre-existing dental problems, neither was taking any medication for at least 2 months prior to this study nor had a smoking history. Anamnesis was performed, as well as clinical examination and a score for bleeding on probing, probing depth, and clinical attachment loss at six sites per tooth, by the periodontist, as described previously ${ }^{18}$. Radiographic examination and occlusion movement data were also obtained and finally the patient was definitely diagnosed as presenting periodontal disease. First, upon clinical examination, the patient did not present bleeding on probing or probing depth $\geq 3 \mathrm{~mm}$ (Figure 1 ); however, an extensive clinical attachment loss ranging from one third to two thirds of periodontal support was verified in both arches, as well as in anterior and posterior teeth, which was confirmed by full-mouth radiographic survey (Figure 2 ). The patient also showed gingival recession of 1-3 mm affecting mainly facial tooth surfaces (Figure 1) and some teeth presented abnormal mobility. However, despite this broad attachment and bone loss, the patient presented satisfactory oral hygiene, with no apparent plaque formation or accumulation of gross calculus and the absence of gingivitis or other detectable manifestations of gingival inflammation (Figure 1). It is of note that this patient had never used tobacco products and reported vigorous and intensive toothbrushing, ranging from 5 up to 8 times a day. Moreover, regarding the dental occlusion conditions, no premature or abnormal dental or restoration contact on occlusion contacts
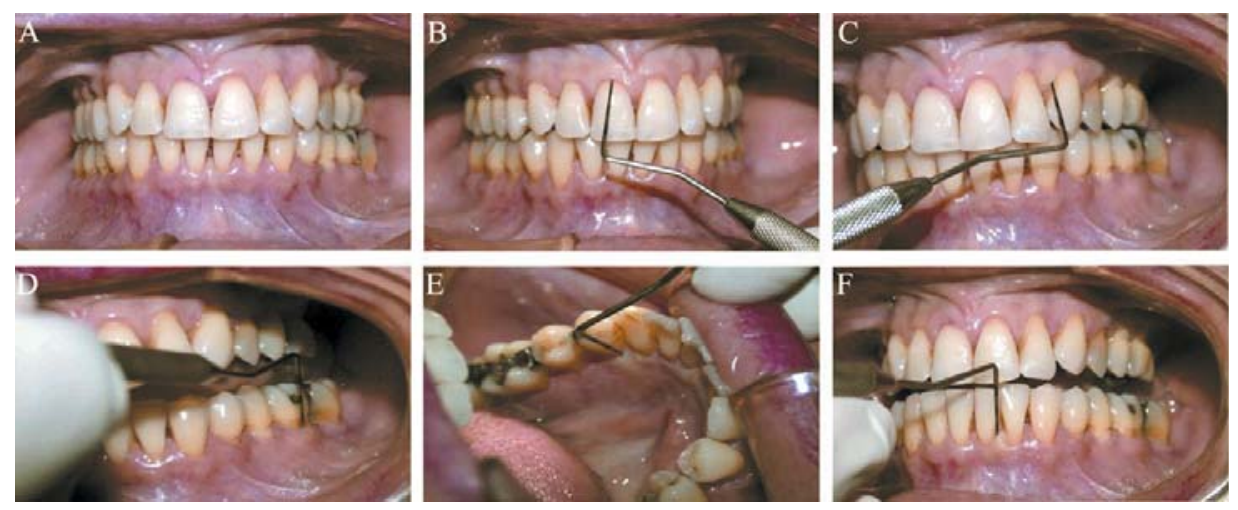

Figure 1- Clinical features of the non-inflammatory destructive periodontal disease (NIDPD) case. Periodontal tissues were evaluated for determination of probing depth, bleeding on probing and gingival inflammation. Satisfactory oral hygiene, with no apparent plaque formation, accumulation of gross calculus or gingivitis was observed (A). Absence of bleeding on probing, absence $\geq 3 \mathrm{~mm}$ probing depths, and the presence of localized gingival recession are depicted in (B-F) 
were verified, as well no laterality or protrusion movements were found to be defective (Figure 3 ).

The patient was questioned about the presence of systemic conditions that would require physician consult, impact on the oral health or influence on periodontal status, but no significant findings were revealed and she reported to be generally in good health, as confirmed by her physician. Nevertheless, complementary exams were still required for deeper investigation, and the patient was submitted to standard protocols for evaluation of hematological, immunological and endocrine markers. Results from complete blood count demonstrate a minor leukopenia (in special neutropenia) and lymphocytosis, both considered non significant by her physician (data not shown). Additionally, the levels of total hydroxyproline, ALP, calcium, calcitonin, urine calcium, PTH (parathyroid hormone), DHEA (dehydroepiandrosterone), FSH (follicle-stimulating hormone), LH (luteinizing hormone), glycemia, total cholesterol, HDL (highdensity lipoprotein), LDL (low-density lipoprotein), triglycerides, type I urine, CRP (C reactive protein) and bone densitometry were completely normal.

To deeper explore the local modification of periodontal status, the biofilm samples obtained from the patient periodontal crevice/pocket were analyzed by Real-time PCR for periodontopathogens detection. The gingival crevicular fluid and biofilm samples from the periodontal crevice/pocket were collected with sterile paper point ISO \#40 (Dentsply, DeTrey, Konstanz, Germany) from at least 5 different bone loss sites in the inferior and superior arches, before treatment recommendations, as described previously ${ }^{15}$. Subgingival biofilm samples were used for PCR assays for periodontopathogens detection. Bacterial or viruses DNA was extracted as previously described ${ }^{15}$, and submitted to Realtime PCR analyses using SybrGreen (Invitrogen, Carlsbad, CA, USA) system, specific primers (the primer sequences are depicted in Figure 4) and $5 \mathrm{ng}$ of DNA in each reaction. The positivity of bacteria or viruses detection in each sample was determined based on the comparison with positive and negative controls. As expected due to the oral hygiene condition of the patient, no positivity was observed for Aggregatibacter actinomycetemcomitans, Porphyromonas gingivalis, Fusobacterium nucleatum or Treponema denticola, in any of the sites evaluated from both the inferior or superior arches. Similarly, there was no DNA detection for viruses related to periodontal diseases like Epstein-Barr virus type 1 (EBV-1), Herpes Simplex virus type 1 (HSV-1) or Human Cytomegalovirus (HCMV) in the same sites (Figure 4).

Gingival fluid was used for ELISA and enzymatic

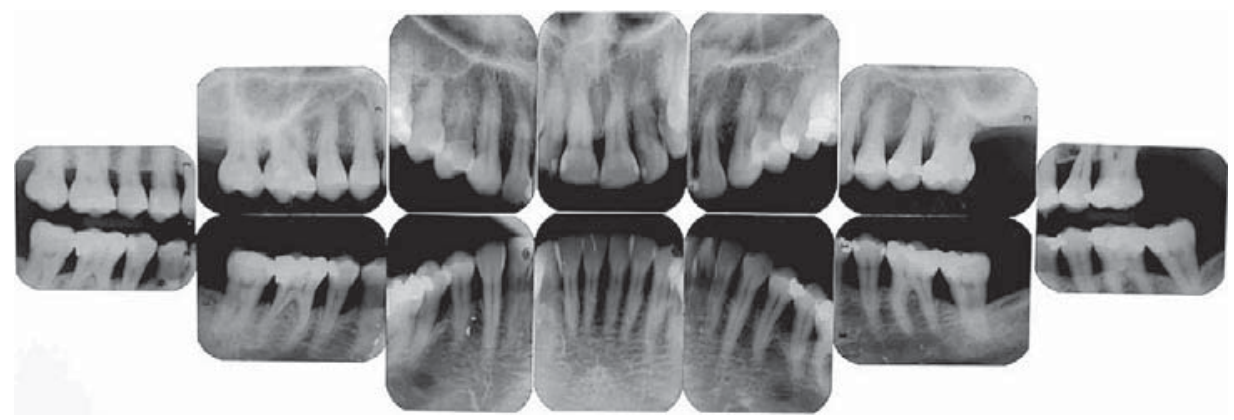

Figure 2- Radiographic features of the non-inflammatory destructive periodontal disease (NIDPD) case. Apparent generalized horizontal alveolar bone loss can be observed in full mouth radiographs

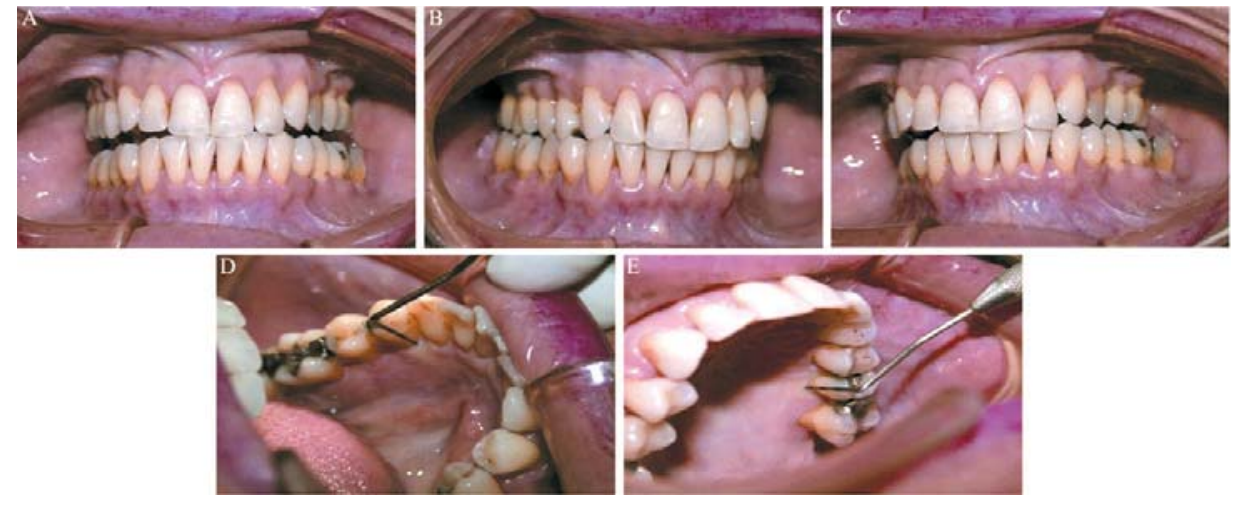

Figure 3- Occlusal features of the non-inflammatory destructive periodontal disease (NIDPD) case. Note absence of defective protrusion $(A)$ or laterality movements $(B, C)$. Occlusion records with no premature or abnormal contacts on occlusion can also be observed (D, E) 


\begin{tabular}{|c|c|c|}
\hline Target & Sense/antisense sequences & Analysis result \\
\hline \multirow[t]{2}{*}{ A.actinomycetemcomitans* } & ATGCCAACTTGACGTTAAAT & \multirow[t]{2}{*}{ negative } \\
\hline & AАACCСАTСTCTGAGTTCTTCT & \\
\hline \multirow[t]{2}{*}{$P$. gingivalis * } & TACCCATCGTCGCCTTGGT & \multirow[t]{2}{*}{ negative } \\
\hline & CGGACTAAAACCGCATACACTT & \\
\hline \multirow[t]{2}{*}{ F. nucleatum* } & GCGGAACTACAAGTGTAGAGGT & \multirow[t]{2}{*}{ negative } \\
\hline & GTTCGACCCCCAACACCTAGTA & \\
\hline \multirow[t]{2}{*}{ T. denticola* } & AGAGCAAGCTCTCCCTTACCGT & \multirow[t]{2}{*}{ negative } \\
\hline & TAAGGGCGGCTTGAAATAATGA & \\
\hline \multirow[t]{2}{*}{ EBV-1* } & CCTGGTCATCCTTTGCCA & \multirow[t]{2}{*}{ negative } \\
\hline & TGCTTCGTTATAGCCGTAGT & \\
\hline \multirow[t]{2}{*}{ HSV-1* } & CGGCCGTGTGACACTATCG & \multirow[t]{2}{*}{ negative } \\
\hline & CTCGTAAAATGGCCССТCC & \\
\hline \multirow[t]{2}{*}{$\mathrm{HCMV}^{*}$} & TGAGCCCGGCGGTGGT & \multirow[t]{2}{*}{ negative } \\
\hline & AGCTCACCGATCACAGACAC & \\
\hline \multirow[t]{2}{*}{ IL1B-3954 SNP } & CTCAGGTGTCCTCGAAGAAATC & \multirow{2}{*}{$\begin{array}{l}\text { non polymorphic } \\
\text { (CC) }\end{array}$} \\
\hline & GCTTTTTTGCTGTGAGTCCCG & \\
\hline \multirow[t]{2}{*}{ TNFA-308 SNP } & AGGCAATAGGTTTTGAGGGCCA & \multirow{2}{*}{$\begin{array}{l}\text { polymorphic } \\
\text { (AA) }\end{array}$} \\
\hline & TCCTCCCTGCTCCGATTCCG & \\
\hline \multirow[t]{2}{*}{ IL6-174 SNP } & TTGTCAAGACATGCCAAGTGCT & \multirow{2}{*}{$\begin{array}{l}\text { polymorphic } \\
\text { (GC) }\end{array}$} \\
\hline & GCCTCAGAGACATCTCCAGTCC & \\
\hline \multirow[t]{2}{*}{ IL10-592 SNP } & GGTCTCTGGGCCTTAGTTTCC & \multirow{2}{*}{$\begin{array}{c}\text { non polymorphic } \\
\text { (CC) }\end{array}$} \\
\hline & AACTTTAGACTCCAGCCACAGA & \\
\hline \multirow[t]{2}{*}{ TGFB1-509 SNP } & TTTTGCCATGTGCCCAGTAG & \multirow{2}{*}{$\begin{array}{l}\text { polymorphic } \\
(\mathrm{CT})\end{array}$} \\
\hline & CACCAGAGAAAGAGGACCAG & \\
\hline \multirow[t]{2}{*}{ MMP1-1607 SNP } & TCGTGAGAATGTCTTCCCATT & \multirow{2}{*}{$\begin{array}{c}\text { polymorphic } \\
(1 \mathrm{G} / 2 \mathrm{G})\end{array}$} \\
\hline & TCTTGGATTGATTTGAGATAAGT & \\
\hline HLA-DR4 (0404) & & positive \\
\hline HLA-B27 & & positive \\
\hline
\end{tabular}

Figure 4- Primer sequences and results from the genetic and microbiological analysis. *Results are representative of both inferior and superior arches samples

tests for quantification of the bone metabolism markers acid phosphatase, alkaline phosphatase and RANKL, and inflammatory cytokines IL-1 $\beta$ and TNF- $\alpha$, performed as previously described ${ }^{39}$. These results were compared to 12 control and 12 chronic periodontitis subjects who also had their gingival fluid collected from healthy or diseased sites respectively, as selected before ${ }^{9,15,55}$. Although systemic bone metabolism markers were in the normality levels, we observed elevated production of $A C P, A L P$ and RANKL in the gingival crevicular fluid (GCF) samples, in concentration similar to those found in chronic periodontitis patients (Figures 5AC). Conversely, the levels of IL-1 $\beta$ and TNF-a were found to be similar in the samples collected from the patient and the controls subjects, while higher levels of inflammatory mediators were detected in the chronic periodontitis sites (Figures $5 \mathrm{~A}-\mathrm{C}$ ). These results suggested that, although systemic features were not altered, the local bone metabolism was indeed modified, as observed when periodontal sites were evaluated.

In order to verify whether features like HLA or genetic polymorphisms could also accomplish for the extensive bone loss observed, the patient was typed for HLA molecules and single nucleotide polymorphisms (SNPs) in gene coding for cytokines associated to periodontal disease susceptibility, as described previously $5,6,9,15,47,48,55$. We also verified if the genetic status of the patient could account for the differential clinical condition observed. When the IL-1ß 3954 SNP was analyzed, we observed the non-polymorphic CC genotype. The present data also demonstrated that the patient carried the IL6-174 GC genotype (polymorphic heterozygous), the AA polymorphic genotype for TNFA-308 SNP, 
TNF- $\alpha$

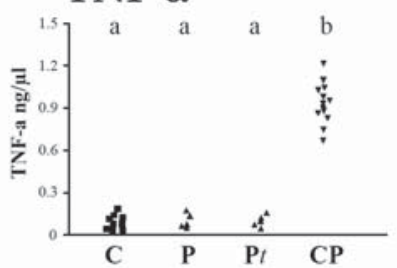

$\mathrm{ACP}$
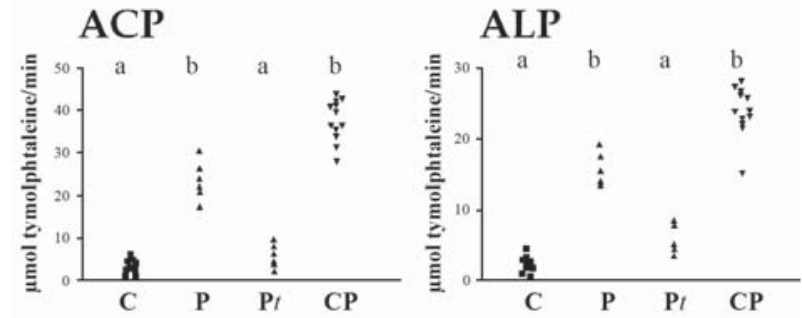

\section{RANKL}

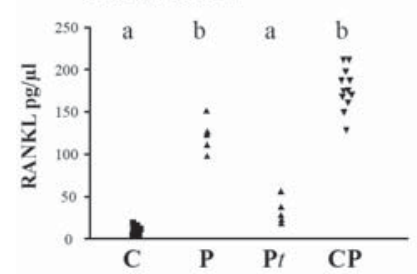

Figure 5- Quantification of inflammatory cytokines and bone metabolism mediators in non-inflammatory destructive periodontal disease. IL-1 $\beta$, TNF- $\alpha$, acid phosphatase (ACP), alkaline phosphatase (ALP) and RANKL were quantified in the gingival crevicular fluid of non-inflammatory destructive periodontal disease $(\mathrm{N}=5$ sites) patient before $(\mathrm{P})$ and after $(\mathrm{Pt})$ treatment recommendations (i.e. a less vigorous and diminished frequency of toothbrushing). Data were compared to control healthy sites $(\mathrm{C} ; \mathrm{N}=12)$ and chronic periodontitis sites ( $\mathrm{CP} ; \mathrm{N}=12$ ) from distinct patients, and analyzed by one-way ANOVA followed by Bonferroni's post test, different letters indicate the statistically significant differences $(P<0.05)$

the MMP1-1607 - genotype 1G/2G (polymorphic heterozygous), the non-polymorphic CC genotype of IL-10 592 SNP and TGFB1 -509 - genotype CT (polymorphic heterozygous) (Figure 4). The genetic analysis also demonstrated that the patient was positive for HLA-DR4 (*0404) and HLA-B27 alleles, described to be associated with bone resorptive diseases like rheumatoid arthritis and periodontal diseases. Interestingly, the patient reported a familial history of rheumatoid arthritis too.

Altogether, the data obtained in the present case showed that the periodontal disease presented by the patient was not a direct consequence of the usual causes like microbiological factors. Instead, it could involve genetic findings and, in special, periodontal trauma possibly due to excessive toothbrushing and oral hygiene, similar to some gingival recession cases. In view of these results, a less vigorous and diminished frequency of toothbrushing was recommended to the patient. At 2- and 6-month re-evaluations, the clinical periodontal condition, including bone loss was stabilized. Five years of follow-up revealed that the clinical scenario was stable without further evidence of disease progression, since no redness or bleeding on probing, increased probing depth or attachment loss, or tooth mobility were observed. In fact, the clinical images and the panoramic radiography (Figure 6), after 5 years of follow-up, showed no progression in the generalized horizontal alveolar bone loss when compared to the initial findings. Moreover, after controlling the excessive toothbrushing, the levels of ALP, ACP and RANKL in gingival crevicular fluid in diseased sites were also greatly reduced, reaching nearby the control levels (Figures 5A-C).

\section{DISCUSSION}

Periodontal diseases are usually recognized as infectious diseases in which an unbalanced host immune response to periodontopathogens leads to bone resorption and tooth loss ${ }^{10,21,22}$. As this is a disease caused by accumulation of dental plaque, it is largely accepted that poor hygiene habits corroborate to the disease development, as well as genetic and immunologic factors, besides external factors like smoking $10,21,22$. In contrast, the etiology of gingival recession, that is the exposure of the root surface due to apical migration of the gingival margin, may involve anatomical and iatrogenic factors like excessive toothbrushing and periodontal trauma besides the association with gingivitis or periodontitis ${ }^{32}$. In this sense, in spite of inappropriate toothbrushing being a risk factor for gingival recession, oral hygiene is believed to be the main preventive action to avoid periodontitis development in susceptible individuals. The study described here showed an atypical case in which bone loss occurred in a patient with good oral hygiene conditions, who had never smoked, did not present apparent plaque formation or gross calculus and was absent of visible gingival inflammation. These unusual clinical presentation intrigued us to deeper investigate the microbiological, immunological and genetic factors that could be involved in this rare case of NIDPD.

We initially investigated the overall systemic condition of the patient, in order to rule out possible systemic alterations that could be a trigger for alveolar bone loss. However, no significant immunological, hematological or endocrine alterations were found. Of special interest in this case, bone metabolism markers hydroxyproline, ALP, calcium, calcitonin, urine calcium ${ }^{13}$ were investigated. However, once again, no alterations were found in these factors levels. In addition, bone densitometry analysis 

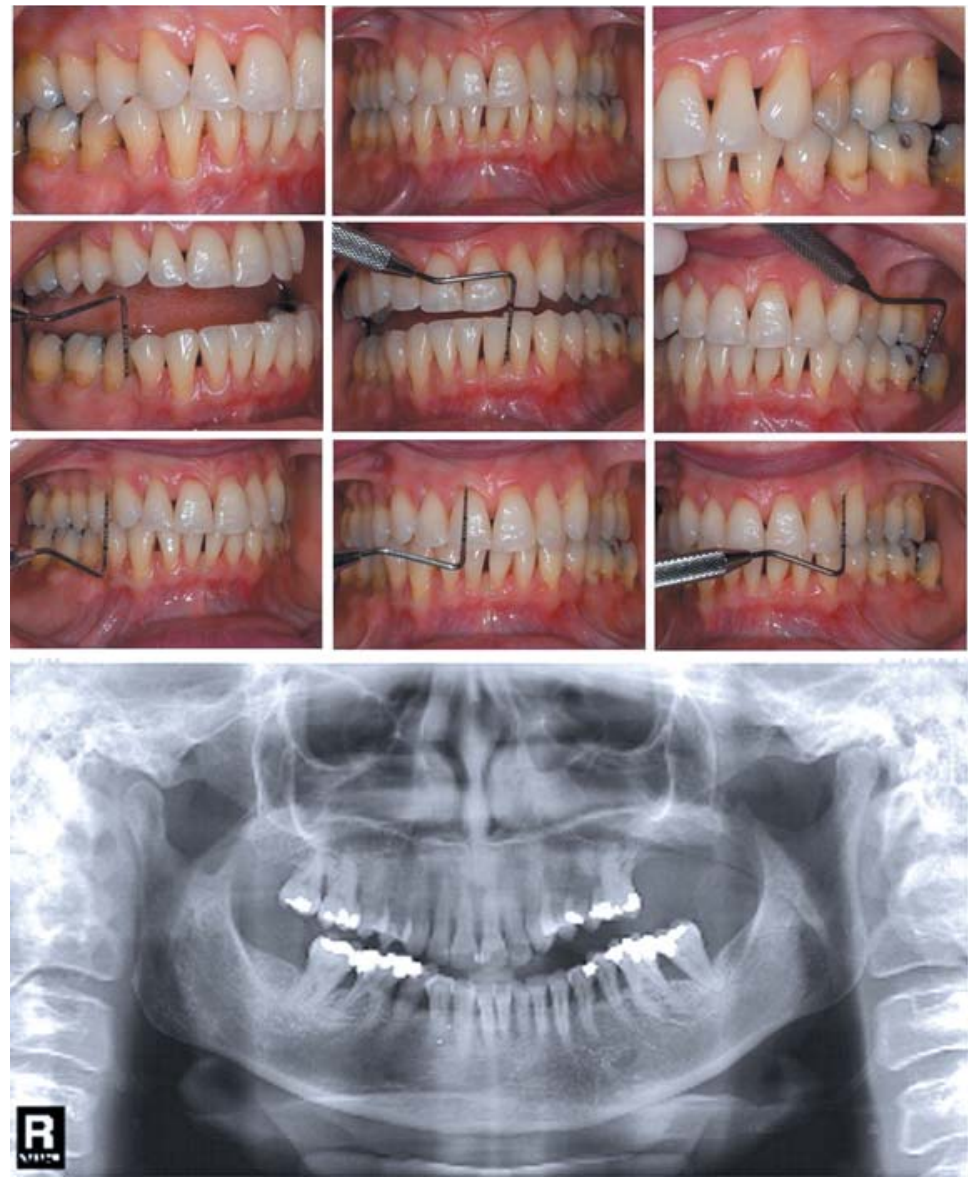

Figure 6- Clinical and radiographic features of the non-inflammatory destructive periodontal disease (NIDPD) case (5 years follow-up). Satisfactory oral hygiene, with no apparent plaque formation, accumulation of gross calculus or gingivitis was observed. Absence of bleeding on probing, absence of $\geq 3 \mathrm{~mm}$ probing depths, and the presence of localized gingival recession are also depicted. The alveolar bone status concerning the generalized horizontal alveolar bone loss can be observed and considered stable when compared with initial radiographic analysis

results were completely normal.

Concerning oral evaluation, the dimensions of the gingival tissue and of the different parts of the masticatory mucosa have become a subject of considerable interest and controversy in periodontics regarding the epidemiologic and the therapeutic point of view. Probably due to a thin and narrow keratinized gingival band a patient is possibly more vulnerable to traumatic injury, leading to a greater tendency of developing gingival recessions, if compared to subjects presenting a thicker keratinized tissue. Nevertheless, the patient showed a satisfactory gingival phenotype and even if that was not the case, an additional stimulatory factor, such as, inflammatory response or a mechanical trauma, is necessary to trigger alveolar bone resorption ${ }^{16}$. Therefore, our next step was to investigate bone resorption markers in gingival crevicular fluid (GCF) of the patient, comparing the values with sites presenting "classical" chronic periodontitis or periodontal health to confirm the occurrence of active bone resorption. The analysis demonstrated the presence of high levels of ACP, ALP and RANKL in the multiple GCF samples collected, in levels comparable with those found in chronic periodontitis sites. RANKL is the main stimulatory factor for the differentiation and activation of osteoclasts ${ }^{4,29}$ and its high expression could justify the extensive bone loss presented by the patient. Indeed, high RANKL levels have been associated with active bone loss in periodontal sites ${ }^{12,18,36}$. While ALP is involved in the mineralization process and is an important marker of osteoblast maturation ${ }^{11,13,49}$, ACP is implicated in bone resorption ${ }^{42}$. In this study, both ALP and ACP levels were increased in the gingival crevicular fluid of the patient during the intensive oral hygiene care period and bone lesion, suggesting an elevated bone metabolism in this sites. Recent studies showed that bone resorption can trigger bone formation, in a process called coupled bone formation, and that the occurrence of high levels of both bone resorption and formation markers can be associated with this process ${ }^{34}$. Interestingly, during orthodontic tooth movement, even the pressure side, characterized by the predominance of bone resorption activity, bone formation markers can 
also be detected ${ }^{19,20}$. However, under pathological conditions, local alterations (still poorly known) impair the subsequent bone formation ${ }^{3,34}$ which we believe to happen in the clinical situation presented herein.

To investigate the mechanisms underlying the active bone resorption in the absence of clinical signs of periodontal infection or inflammation, we next inspected subgingival samples for the presence of periodontopathogens commonly found in the diseased sites. No positivity was observed for specific periodontopathogens (Aggregatibacter actinomycetemcomitans, Porphyromonas gingivalis, Fusobacterium nucleatum and Treponema denticola) or for certain viruses (HCMV, EBV-1 and HSV-1) also known to be present in human periodontitis lesions ${ }^{22,45,46}$. These initial results suggested that in this case the clinical outcome of periodontal disease was probably not dependent on periodontopathogens.

As microbiological data did not clarify the pathogenesis of this special case, we proceed to the genetic and immunological analysis of the patient profile. Interestingly, no evidences of inflammatory activity were found at immunological investigation; since the levels of IL-1 $\beta$ and TNF-a in all the sites evaluated were compatible with those seen in clinically healthy periodontal tissues, and significantly lower than those from chronic periodontitis sites. Then, to characterize a putative genetic background prone to the development of bone loss, we next investigated some single nucleotide polymorphisms (SNP) that could characterize a susceptibility phenotype. Interestingly, both potential protective and risk alleles/genotypes were identified. The patient presented the non-polymorphic CC genotype of IL-1b 3954 SNP, not associated with IL-1 $\beta$ increase in periodontitis patients who carries the $T$ allele ${ }^{15}$. The patient was also evaluated regarding IL6-174 polymorphism, previously associated with susceptibility to chronic periodontitis in Brazilian patients ${ }^{55}$ and the resistance $C$ allele (GC heterozygote genotype) was found. Concerning the IL10-592 SNP, the patient was found to carry the CC genotype, associated with high production of the anti-inflammatory cytokine IL-10, and therefore considered a protective genotype regarding bone loss $^{9}$. On the other hand, half of the investigated SNPs suggest the occurrence of risk alleles for bone loss. The AA polymorphic genotype for TNFA-308 SNP, presented by the patient, is related to increased TNF-a production and periodontitis severity ${ }^{55}$. The patient also carried the CT heterozygous genotype of the -509TGFB1 SNP, in which the T allele seems to confer a greater risk for the development of periodontal disease ${ }^{48}$. In addition, regarding the MMP1-1607 SNP, the patient presented the polymorphic heterozygous genotype $1 \mathrm{G} / 2 \mathrm{G}$. The MMP-1 2G allele was previously associated with severe chronic periodontitis in Brazilian subjects ${ }^{47}$ and to increase MMP-1 mRNA transcription ${ }^{43}$, and therefore this allele could be considered an additional risk factor for bone loss.

However, it is important to remind that all these SNPs cited above have been associated with "classical" infectious and inflammatory pathogenesis of periodontal disease, and may not be relevant to this particular case in the view of the distinct non-inflammatory characteristics observed. Interestingly, periodontitis and arthritis have been found to be clinically associated and to share several characteristics such as the chronic nature of the inflammatory reaction associated with bone resorption activity, suggesting that these diseases could share genetic susceptibility/resistance patterns ${ }^{2,30,37}$. Conversely to the classic inflammatory SNPs, HLA analyses have been demonstrated to be useful to access putative susceptibility to the development of bone loss even under non-infectious non-inflammatory circumstances. The HLA analysis showed that the patient carried the HLA-DR4 (*0404) and HLA-B27 alleles. The HLA-DR4 subtypes 0401, 0404, 0405 and 0408 are frequently found in severe periodontitis patients and these alleles can also be considered risk factors for rapidly progressive periodontitis ${ }^{6}$. Moreover, a genetic marker for susceptibility to rheumatoid arthritis is carried by the highly polymorphic HLA-DRB1 locus and some alleles coding for a conserved linear sequence of amino acids in the DRb1 chain of the HLA-DRa/b heterodimer (including QRRAA in *0404) referred to as the "shared epitope" are also a severity marker for periodontal bone destruction in rheumatoid arthritis $^{33}$. On the other hand, regarding HLA and bone metabolism, it was described that HLA-B27 transgenic rats present accelerated alveolar bone loss as an age-dependent process ${ }^{35}$, have bone fragility due to increased bone resorption ${ }^{41}$ and show decreased bone strength when compared to control littermates, similar to that seen in some bone disorders ${ }^{1}$. Indeed, HLA-B27 have been associated with bone disorders in humans ${ }^{7,26}$. These results are in agreement with ours, which showed a patient with atypical bone loss carrying HLA-B27 alleles. However, in the view of the rare occurrence and description in the literature ${ }^{40}$ of non-inflammatory destructive periodontal disease (NIDPD), it is not possible to certainly associate the genetic findings described above with the disease development, and further studies are required to support stronger statements with this matter.

Irrespective of the complete elucidation of the exact pathogenesis of NIDPD, a less vigorous and diminished frequency of toothbrushing was recommended to the patient, based on the treatment applied in the two previously described cases $^{40}$. After 2- and 6-month re-evaluations, 
the clinical periodontal condition, primarily the alveolar bone loss, was stabilized. In addition, this finding was supported by laboratorial analysis, which demonstrate that the levels of ALP, ACP and RANKL in GCF were also greatly reduced, reaching nearby the control levels. Interestingly, studies demonstrate that the force and frequency of toothbrushing can affect the activation and proliferation of cells in the periodontal environment, however such studies were limited to epithelial cells and gingival fibroblasts $24,44,51,52,54$. Concerning the mechanical properties of bone cells, recent studies have demonstrated that these cells are sensitive to mechanotransduction, and that different types of forces may result in biological response, including bone resorption ${ }^{25,27}$. A classic example of forceinduced bone resorption is the orthodontic tooth movement, where compressive forces are associated with bone resorption activity ${ }^{23,31}$. Accordingly, two recent study demonstrate the differential tissue response to the application of forces of distinct magnitude over periodontal tissues ${ }^{55,56}$. While mechanical stimulation provided by $0.02 \mathrm{~N}$ forces accelerated the healing of gingival inflammation by reducing the infiltration of polymorphonuclear leukocytes and enhancing fibroblast proliferation and collagen synthesis ${ }^{14}, 20$ to $40 \mathrm{~N}$ loading resulted in osteoclast recruitment to the periodontal tissues in a force- and time-dependent manner ${ }^{38}$. Therefore, it seems reasonable to assume that mechanotransduction of intense and frequent toothbrushing can result in a response of bone cells; a hypothesis supported by the fact that a lower degree mechanical stimulation (i.e. a less vigorous and diminished frequency of toothbrushing) resulted in reduced levels of bone resorption makers and stabilization of bone loss progression.

The unusual case presented here are compatible with a previous description of non-infectious noninflammatory destructive periodontal disease ${ }^{40}$, in which outcome and progression of periodontal disease constitute a complex and not fully elucidated process. Our clinical, microbiological, immunological and genetic results suggest that a possible combination of excessive force and frequency of mechanical stimulation with a potentially bone loss prone genotype can result in the alveolar bone loss seem in NIDPD. This knowledge may serve as a basis for development of more effective strategies for prevention, diagnosis and treatment of unusual periodontitis form such as NIDPD.

\section{ACKNOWLEDGMENTS}

The authors acknowledge Cristiane Maria Milanezi, Daniele Santi Ceolin and Patricia de Sá Mortagua Germino for her excellent technical assistance. This work was supported by grants from

\section{FAPESP and CNPq.}

\section{REFERENCES}

1- Akhter MP, Jung LK. Decreased bone strength in HLA-B27 transgenic rat model of spondyloarthropathy. Rheumatology (Oxford). 2007;46(8):1258-62.

2- Al-Katma MK, Bissada NF, Bordeaux JM, Sue J, Askari AD. Control of periodontal infection reduces the severity of active rheumatoid arthritis. J Clin Rheumatol. 2007;13(3):134-7.

3- Andersen TL, Sondergaard TE, Skorzynska KE, Dagnaes-Hansen F, Plesner TL, Hauge EM, et al. A physical mechanism for coupling bone resorption and formation in adult human bone. Am J Pathol. 2009;174(1):239-47.

4- Arai F, Miyamoto T, Ohneda O, Inada T, Sudo T, Brasel K, et al. Commitment and differentiation of osteoclast precursor cells by the sequential expression of c-Fms and receptor activator of nuclear factor kappaB (RANK) receptors. J Exp Med. 1999;190(12):174154.

5- Bonfigurelioli R, Conde RA, Sampaio-Barros PD, LouzadaJunior P, Donadi EA, Bertolo MB. Frequency of HLA-B27 alleles in Brazilian patients with psoriatic arthritis. Clin Rheumatol. 2008;27(6):709-12.

6- Bonfil J], Dillier FL, Mercier P, Reviron D, Foti B, Sambuc R, et al. A "case control" study on the role of HLA DR4 in severe periodontitis and rapidly progressive periodontitis. Identification of types and subtypes using molecular biology (PCR.SSO). J Clin Periodontol. 1999;26(2):77-84.

7- Brown MA, Wordsworth BP, Reveille JD. Genetics of ankylosing spondylitis. Clin Exp Rheumatol. 2002;20(6 Suppl 28):S43-9.

8- Caffesse RG, Nasjleti CJ, Kowalski CJ, Castelli WA. The effect of mechanical stimulation on the keratinization of sulcular epithelium. J Periodontol. 1982;53(2):89-92.

9- Claudino M, Trombone AP, Cardoso CR, Ferreira SB Jr, Martins W Jr, Assis GF, et al. The broad effects of the functional IL-10 promoter-592 polymorphism: modulation of IL-10, TIMP-3, and OPG expression and their association with periodontal disease outcome. J Leukoc Biol. 2008;84(6):1565-73.

10- Cochran DL. Inflammation and bone loss in periodontal disease. J Periodontol. 2008;79(8 Suppl):1569-76.

11- Crippa GE, Beloti MM, Cardoso CR, Silva JS, Rosa AL. Effect of growth hormone on in vitro osteogenesis and gene expression of human osteoblastic cells is donor-age-dependent. J Cell Biochem. 2008;104(2):369-76.

12- Crotti T, Smith MD, Hirsch R, Soukoulis S, Weedon H, Capone $\mathrm{M}$, et al. Receptor activator NF kappaB ligand (RANKL) and osteoprotegerin (OPG) protein expression in periodontitis. J Periodontal Res. 2003;38(4):380-7.

13- Delmas PD. Biochemical markers of bone turnover. J Bone Miner Res. 1993;8(Suppl 2):S549-55.

14- Ekuni D, Yamanaka R, Yamamoto T, Miyauchi M, Takata T, Watanabe T. Effects of mechanical stimulation by a powered toothbrush on the healing of periodontal tissue in a rat model of periodontal disease. J Periodontal Res. 2010;45(1):45-51.

15- Ferreira SB Jr, Trombone AP, Repeke CE, Cardoso CR, Martins W Jr, Santos CF, et al. An interleukin-1beta (IL-1beta) singlenucleotide polymorphism at position 3954 and red complex periodontopathogens independently and additively modulate the levels of IL-1beta in diseased periodontal tissues. Infect Immun. 2008;76(8):3725-34.

16- Fu JH, Yeh CY, Chan HL, Tatarakis N, Leong DJ, Wang HL. Tissue biotype and its relation to the underlying bone morphology. J Periodontol. 2010;81(4):569-74.

17- Garlet GP, Cardoso CR, Campanelli AP, Ferreira BR, AvilaCampos MJ, Cunha FQ, et al. The dual role of p55 tumour necrosis factor-alpha receptor in Actinobacillus actinomycetemcomitansinduced experimental periodontitis: host protection and tissue destruction. Clin Exp Immunol. 2007;147(1):128-38. 
18- Garlet GP, Martins W Jr, Fonseca BA, Ferreira BR, Silva JS. Matrix metalloproteinases, their physiological inhibitors and osteoclast factors are differentially regulated by the cytokine profile in human periodontal disease. J Clin Periodontol. 2004;31(8):671-9. 19- Garlet TP, Coelho U, Repeke CE, Silva JS, Cunha FQ, Garlet GP. Differential expression of osteoblast and osteoclast chemmoatractants in compression and tension sides during orthodontic movement. Cytokine. 2008;42(3):330-5.

20- Garlet TP, Coelho U, Silva JS, Garlet GP. Cytokine expression pattern in compression and tension sides of the periodontal ligament during orthodontic tooth movement in humans. Eur J Oral Sci. 2007;115(5):355-62.

21- Graves D. Cytokines that promote periodontal tissue destruction. J Periodontol. 2008;79(8 Suppl):1585-91.

22- Haffajee AD, Socransky SS. Microbiology of periodontal diseases: introduction. Periodontol 2000. 2005;38:9-12.

23- Henneman S, Von den Hoff JW, Maltha JC. Mechanobiology of tooth movement. Eur J Orthod. 2008;30(3):299-306.

24- Horiuchi M, Yamamoto T, Tomofuji T, Ishikawa A, Morita $M$, Watanabe T. Toothbrushing promotes gingival fibroblast proliferation more effectively than removal of dental plaque. J Clin Periodontol. 2002;29(9):791-5.

25- Iqbal J, Zaidi M. Molecular regulation of mechanotransduction. Biochem Biophys Res Commun. 2005;328(3):751-5.

26- Khan MA. HLA-B27 and its pathogenic role. J Clin Rheumatol. 2008;14(1):50-2.

27- Klein-Nulend J, Bacabac RG, Mullender MG. Mechanobiology of bone tissue. Pathol Biol (Paris). 2005;53(10):576-80.

28- Kornman KS. Mapping the pathogenesis of periodontitis: a new look. J Periodontol. 2008;79(8 Suppl):1560-8.

29- Kotake S, Udagawa N, Takahashi N, Matsuzaki K, Itoh K, Ishiyama $\mathrm{S}$, et al. IL-17 in synovial fluids from patients with rheumatoid arthritis is a potent stimulator of osteoclastogenesis. J Clin Invest. 1999;103(9):1345-52.

30- Kramer JM, Gaffen SL. Interleukin-17: a new paradigm in inflammation, autoimmunity, and therapy. J Periodontol. 2007; 78(6): 1083-93.

31- Krishnan V, Davidovitch Z. Cellular, molecular, and tissue-level reactions to orthodontic force. Am J Orthod Dentofacial Orthop. 2006;129(4):469.e1-32.

32- Litonjua LA, Andreana S, Bush PJ, Cohen RE. Toothbrushing and gingival recession. Int Dent J. 2003;53(2):67-72.

33- Marotte $H$, Farge $P$, Gaudin $P$, Alexandre $C$, Mougin B, Miossec $P$. The association between periodontal disease and joint destruction in rheumatoid arthritis extends the link between the HLA-DR shared epitope and severity of bone destruction. Ann Rheum Dis. 2006;65(7):905-9.

34- Martin T, Gooi JH, Sims NA. Molecular mechanisms in coupling of bone formation to resorption. Crit Rev Eukaryot Gene Expr. 2009;19(1):73-88.

35- May NY, Tatakis DN. Accelerated alveolar bone loss in male HLA-B27 transgenic rats: adult onset. J Periodontal Res. 2004;39(1):33-6.

36- Menezes R, Garlet TP, Letra A, Bramante CM, Campanelli AP, Figueira RC, et al. Differential patterns of receptor activator of nuclear factor kappa B ligand/osteoprotegerin expression in human periapical granulomas: possible association with progressive or stable nature of the lesions. J Endod. 2008;34(8):932-8.

37- Mercado FB, Marshall RI, Klestov AC, Bartold PM. Relationship between rheumatoid arthritis and periodontitis. J Periodontol. $2001 ; 72(6): 779-87$.

38- Nozaki K, Kaku M, Yamashita Y, Yamauchi M, Miura H. Effect of cyclic mechanical loading on osteoclast recruitment in periodontal tissue. 2010;45(1):8-15.

39- Oliveira RR, Schwartz-Filho HO, Novaes AB, Garlet GP, Souza RF, Taba M, et al. Antimicrobial photodynamic therapy in the nonsurgical treatment of aggressive periodontitis: cytokine profile in gingival crevicular fluid, preliminary results. ] Periodontol. 2009;80(1):98-105.
40- Page RC, Sturdivant EC. Noninflammatory destructive periodontal disease (NDPD). Periodontol 2000. 2002;30:24-39. 41- Papet I, El Yousfi M, Godin JP, Mermoud AF, Davicco MJ, Coxam $\checkmark$, et al. HLA-B27 rats develop osteopaenia through increased bone resorption without any change in bone formation. J Musculoskelet Neuronal Interact. 2008;8(3):251-6.

42- Piattelli A, Scarano A, Piattelli M. Detection of alkaline and acid phosphatases around titanium implants: a light microscopical and histochemical study in rabbits. Biomaterials. 1995;16(17):1333-8. 43- Repeke CE, Trombone AP, Ferreira SB Jr, Cardoso CR, Silveira EM, Martins W Jr, et al. Strong and persistent microbial and inflammatory stimuli overcome the genetic predisposition to higher matrix metalloproteinase-1 (MMP-1) expression: a mechanistic explanation for the lack of association of MMP1-1607 singlenucleotide polymorphism genotypes with MMP-1 expression in chronic periodontitis lesions. J Clin Periodontol. 2009;36(9):72638.

44- Sakamoto T, Horiuchi M, Tomofuji T, Ekuni D, Yamamoto T, Watanabe T. Spatial extent of gingival cell activation due to mechanical stimulation by toothbrushing. J Periodontol. $2003 ; 74(5): 585-9$.

45- Saygun I, Kubar A, Ozdemir A, Yapar M, Slots J. Herpesviralbacterial interrelationships in aggressive periodontitis. J Periodontal Res. 2004;39(4):207-12.

46- Socransky SS, Haffajee AD. Periodontal microbial ecology. Periodontol 2000. 2005;38:135-87.

47- Souza AP, Trevilatto PC, Scarel-Caminaga RM, Brito RB, Line SR. MMP-1 promoter polymorphism: association with chronic periodontitis severity in a Brazilian population. J Clin Periodontol. 2003;30(2):154-8.

48- Souza AP, Trevilatto PC, Scarel-Caminaga RM, Brito RB, Line SR. Analysis of the TGF-beta1 promoter polymorphism (C-509T) in patients with chronic periodontitis. J Clin Periodontol. 2003;30(6):519-23.

49- Spolidorio LC, Nassar PO, Nassar CA, Spolidorio DM, Muscara MN. Conversion of immunosuppressive monotherapy from cyclosporin a to tacrolimus reverses bone loss in rats. Calcif Tissue Int. 2007;81(2):114-23.

50- Tanaka M, Hanioka T, Kishimoto M, Shizukuishi S. Effect of mechanical toothbrush stimulation on gingival microcirculatory functions in inflamed gingiva of dogs. J Clin Periodontol. $1998 ; 25(7): 561-5$.

51- Tomofuji T, Ekuni D, Yamamoto T, Horiuchi M, Sakamoto T, Watanabe T. Optimum force and duration of toothbrushing to enhance gingival fibroblast proliferation and procollagen type I synthesis in dogs. J Periodontol. 2003;74(5):630-4.

52- Tomofuji T, Morita M, Horiuchi M, Sakamoto T, Ekuni D, Yamamoto $T$, et al. The effect of duration and force of mechanical toothbrushing stimulation on proliferative activity of the junctional epithelium. J Periodontol. 2002;73(10):1149-52.

53- Tomofuji T, Sakamoto T, Ekuni D, Yamamoto T, Watanabe T. Location of proliferating gingival cells following toothbrushing stimulation. Oral Dis. 2007;13(1):77-81.

54- Trevilatto PC, Scarel-Caminaga RM, Brito RB Jr, Souza AP, Line SR. Polymorphism at position -174 of IL- 6 gene is associated with susceptibility to chronic periodontitis in a Caucasian Brazilian population. J Clin Periodontol. 2003;30(5):438-42.

55- Trombone AP, Cardoso CR, Repeke CE, Ferreira SB Jr, Martins W Jr, Campanelli AP, et al. Tumor necrosis factor-alpha -308G/A single nucleotide polymorphism and red-complex periodontopathogens are independently associated with increased levels of tumor necrosis factor-alpha in diseased periodontal tissues. J Periodontal Res. 2009;44(5):598-608.

56- Yamamoto T, Tomofuji T, Ekuni D, Sakamoto T, Horiuchi M, Watanabe T. Effects of toothbrushing frequency on proliferation of gingival cells and collagen synthesis. J Clin Periodontol. $2004 ; 31(1): 40-4$. 